\title{
Quelques cas de surra au Cambodge
}

\author{
par R. ARRIGHI
}

\begin{abstract}
EN novembre 1941, je recevais un lot d'une vingtaine de petites juments de race indigène saisies. Ces animaux avaient été précédemment visités; rien d'anomal a'avail été sigrjalé, Elles devaient être revendues aux éleveurs de la région.

Pendant les quelques jours nécessaires à la construction d'une écurie d'isolement situé à $1 \mathrm{~km}$. 500, ce lot (toutes en bon état apparent et en état de gestation avancé) fut hébergé tant bien que mal. Une autre partie de ce lot avait été dirigée sur Pnom-Penh; une semaine après, le surra est signalé sur celles de Pnom-Penh.

Prise de temperature et examen du sang de l'oreille à l'état frais entre lame et lamelle pour chaque animal : pas de température, pas de trypanosomes.
\end{abstract}

Appelé en toumée dans le nord de mon secteur où je reste quinze jours retenu par une épidémie de peste bovine; à mon retour rien à signaler dans le troupeau de juments, mais par contre, on me rend compte du décès d'un beau taureau de race Ongole âgé de cinq ans. La cause en est attribuée au barbone par le vétérinaire indochinois. L'animal aurait présenté des signes vagues vers midi, le soir à 17 heures un cedeme léger de la gorge; il serait mort dans la nuit malgré une injection sous-cutanée de $120 \mathrm{cc}$. de sérum antibarbone.

Une semaine après, un nouveau taureau âgé de trois ans, de race Ongole, présente les mêmes symptômes, le soir à 17 heures, fièvre $40^{\circ}$, cedème de la gorge gros comme une tête d'enfant. Un examen du sang entre lame et lamelle pratiqué ne révèle pas de trypanosomes.

II reçoit en injection cutanée et en deux points différents, 300 cc. de sérum antibarbone; il meurt néanmoins dans la nuit.

Tous les animaux de la ferme etaient régulièrement vaccinés contre la peste bovine, le charbon bactéridien et le barbone; la dernière vaccination datait de deux mois. Si l'on pouvait être tenté de penser à une défaillance du vaccin antibarbone, it était étonnant que le sérum antibarbone soit lui aussi inefficace, provenant d'un lot différent, alors qu'habituellement on assistait à une véritable résurrection des animaux, quelquefois même en décubitus latéral complet.

Autopsie; œdème gélatineux sous-glossien avec infiltration de liquide légèrement rosé, foie normal, rate peu congestionnée.

Deux jours après, deux des meilleures laitières du troupeau (petites vaches de race cambodgienne, âgées de six ans, en excellent état physique) présentaient un œdème sous-glossien de la grosseur d'une tête d'enfant; peu de température, pas de trypanosomes décelés dans le sang périphérique à l'examen à l'état frais entre lame et lamelle.

II ne pouvait s'agir ni de charbon bactéridien ni de barbone, les fiches de vaccination et les deux cas précédents faisaient foi.

Dans le doute et quoique ces deux vaches soient en lactation et en etat de gestation de quatre ou cinq mois, on leur injecte dans la jugulaire 3 grammes de Naganol en solution au dixième, poussés très lentement.

Laissées à l'étable pendant une semaine, elles ont gardé leur lait, n'ont pas avorté, l'œdème a disparu complètement au bout de ce temps. Une nouvelle injection de 3 grammes de Naganol a été pratiquée douze jours après la première dans les mêmes conditions.

Environ une semaine après, deux nouvelles vaches laitieres aussi parmi les meilleures : une métisse ongole-cambodgienne et une cambodgienne ont présenté le même cedème sous-glossien; traitées de la même façon, on a obtenu le même succès. Malgré l'impossibilité de mettre les trypanosomes en évidence dans le sang circulant, les moyens dont nous disposions étant insuffisants, les preuves therapeutiques paraissaient probantes; tout le troupeau bovin a été traité indistinctement au Naganol aux mêmes doses. Plus aucun accident n'a été déploré.

Les vaches de race Ongole qui, par la suite, montraient un amaigrissement anormal ont été soumises au traitement par le Naganol; seule une Ongole de quatre ans a dû subir trois traitements successifs à trois mois d'intervalle, soit, en tout, 18 grammes de Naganol en intraveineuse en solution au dixième. Elle a repris son embonpoint et, par la suite, a mis bas les deux années suivantes, d'un veau chaque fois, qu'elle a parfaitement nourri.

En même temps que les deux dernières vaches laitières malades, l'étalon « Hang Phi Vang ", bai trois huitièmes arabe, cinq huitièmes annamite, $1 \mathrm{~m}$. 28, âgé de neuf ans, présente de l'inappétence, fièvre $40^{\circ}$, muqueuses gris sale, très nombreux trypanosomes à l'examen du sang périphérique entre lame et lamelle. Des plaques envoyées au Laboratoire de Pnom-Penh confirment le diagnostic de surra. L'animal est traité le soir même par une 
injection de 3 grammes de Naganol au dixièmo on intraveineuse; les trypanosomes ont complètement disparu le lendemain matin. Douze jours après, nouvelle injection de 3 grammes en intraveineuse; l'animal semble en bonne santé, après un repos de trois mois, il est remis à la monte, sa température est surveillée et des examens de sang pratiqués chaque semaine.

Un mois après, il a présenté un léger balancement du train postérieur sans qu'on puisse mettre les trypanosomes en évidence dans le sang périphérique. Traité néanmoins par une nouvelle sêrie de deux injections de Naganol, le balancement n'a fait que s'accentuer pour se transformer en paralysie un mois après. Il est mort sans avoir présenılé d'iclère ou d'œdème.

Début janvier 1942, la jument «Trottinette», gris pommelé, demi-sang arabe, demi-sang annamite, I m. 32, âgée de huit ans, présente de la fièvre et de très nombreux trypanosomes à l'examen' du sang périphérique entre lame et lamelle: Même traitement qu' «Hang Phi Vang "; les trypanosomes disparaissent le lendemain. Surveillee et en bon état, elle est remplie en juin 1942. Sa gestation semble normale. En janvier 1943, elle présente de la température et de très nombreux trypanosomes dans le sang périphérique. Malgré son état de gestation avancé, elle reçoit 3 grammes de Naganol en solution au vingtième, en injection intramusculaire, au poitrail. Elle avorte dans la matinëe sans suites postérieures d'infection utérine, mais elle accuse le lendemain un très volumineux œedème des membres et du périnée; les jours suivants un ictère jaune orangé apparaît, la courbe de température accuse des clochers. En définitive, la jument meurt avec une paralysie du train postérieur, des œedèmes volumineux et un ictère jaune orangé. Autopsie : foie à bords épaissis d'un volume double de la normale et prèsentant des lésions de dégénérescence; cadavre infiltré, œdème rosé, lésions de misère physiologique.

Sur le troupeau initial envoyé de Pnom-Penh en novembre, deux juments sont trouvées avec des trypanosomes en janvier. Immédiatement traitées avec $1 \mathrm{gr} .50$ de Naganol en intraveineuse, elles ont avorté dans les trois heures qui suivaient, sans autres suites fâcheuses. Une nouvelle injection de $1 \mathrm{gr} .50$ est pratiquée douze jours après la première. Dans le mois qui a suivi, dix autres juments ont été reconnues trypanosomées et traitées de la même façon que les deux premières; les autres à poils longs sont restées indemnes et ont termine leur gestation sans encombres. Elles ont été cédées à des éleveurs.

Quant aux juments ayant été infectées, elles ont eu des destins divers; huit de petite taille ont reçu une nouvelle série de deux injections, trois mois après et ont été vendues pour tirer les voitures. J'ai gardé les quatre autres dans l'écurie, strictement isolées.

Après une nouvelle série de deux injections à 1 gr. 50 de Naganol, trois mois après elles ont été remplies d'avril à juin 1942, par un étalon alezan trois huitièmes arabe, cinq huitièmes annamite, de $1 \mathrm{~m}$. 34. Deux ont avorté vers le huitième mois avec des trypanosomes dans le sang, une autre' a présenté des trypanosomes les derniers jours de la gestation, a mis bas; traitée au Naganol par deux injections à $1 \mathrm{gr} .50$, le poulain étant mort dans les vingt-quatre heures ayant suivi sa naissance. La quatrième a mis bas début'avril 1943 un poulain en bon état et l'a élevé. Elle a reçu deux mivis après sa mise bas deux injections de sécurité de l' gr. 50. Resaillie avec succès neuf jours après sa mise bas, elle a eu un nouveau poulain d'excellente venue.' Au 9 mars 1945 elle n'avait plus présenté d'accidents morbides; on n'avait jamais plus décelé de trypanosomes dans la circulation périphérique.

\section{ILE SURRA SUR DES ANIMAUX DE TRAVAIL}

Au Cambodge, conme dans tout le sud indochinois, les grandes plantations de caoutchouc se' sont trouvées aux prises avec une crise de transport très grave. Sur tous les petits parcours interieurs, on a remplacé les camions par des attelages de boufs et par des chevaux. On a constitué d'importants troupeaux de 900 et 1.000 bovins et 50 et 100 chevaux indigènes répartis en plusieurs écuries.

Ties achats ont été faits comme on pouvait, et, au début, toutes les garanties sanitaires n'ont peutêtre pas été prises; d'autre part,' ces plantations sont situées en bordure de la grando forĉt tropicale avec toute la gamme de bovidés et cervidés sauvạges. Le surra est apparu dans certaines écuries: Les chevaux traités avec $1 \mathrm{gr}, 50$ de Naganol en intraveineuse à douze jours d'intervalle étaient groupés par la suite en écuries de suspects. Deux examens mensuels systématiques du sang avec prise de température ont permis de déceler autant que possible les animaux en rechute et de maintenir l'effectif en état assez satisfaisant. Ils étaient soumis à un travail léger sur des parcours isolés; malgré cela, ils étaient sujels à de fréquents accidents de fourbure, des abcès et des plaiẹs multiples; l'effectif d'indisponibles atteignait fréquemment $50 \%$; ceux soumis à un' travail quelque peu pénible rechutaient.

Le sous-directeur d'une des plantations possédait personnellement une jument bai trois quarts arabe, un quart annamite, âgée de cinq ans, $1 \mathrm{~m}$. 38, et trois autres juments annamites de $1 \mathrm{~m}$. 18 à $1 \mathrm{~m}$. 22 âgées de deux ans, deux ans et demi et quatre ans. Ces 
animaux, dans une écurie isolée, vivaient là depuis des années en excellente santé ; la plus jeune y était née. Dans les nouveaux lots se trouvaient quelques sujets indigenes d'assez belle taille; en quelques mois il en acheta cinq au fur et à mesure. Sa jument trois quarts arabe était pleine depuis un mois environ d'un étalon pur sang arabe, lorsqu'elle présenta quelques signes vagues et de l'anorexie. A l'examen du sang, de très nombroux trypanosomes sont décelés, la température atteint 40\%5; conjonctive gris sale.

Trois autres juments de'l'écurie sont trouvées avec des trypanosomes. Elles sont toutes traitées sur-lechamp. La grande a reçu 3 grammes en intraveineuse, lés autres $1 \mathrm{gr}$. 50; mêmes doses douze jours après. La grande n'a pas avorté mais a présenté une très forte réaction œdémateuse de la vulve avec pétéchies, qui a disparu en trois semaines. Trois en état de gestation avancé ont avorté sans autres suites. Les trypanosomes avaient disparu de la circulation générale dés le lendemain. La jument trois quarts arabe soumise chaque décade à un examen hématologique entre lame et lamelle et prise de température s'est maintenue jusqu'à la fin en bon état. La gestation s'est prolongée plus de douze mois; les deux derniers mois, elle a présenté quelques œdèmes des postérieurs qui, en un mois et demi, ont envahi toute la région sous-abdominale et le périnée. A la période des cedèmes onvahissants, elle était mal assurée sur son train postérieur pour être complètement paraplégique quelques jours avant sa mise bas, avec des trypanosomes nombreux dans le sang les derniers jours; la température a accusé des clochers, elle présentait un ictère jaune orangé. L'expérience m'avait montré que, dans ces conditions, pour les animaux ayant une forte proportion de sang importé, il ne fallait rien attendre d'un nouvel essai de traitement. Dans l'état paraplégique où se trouvait la mère, le pronostic était sombre. Les codemes des voies génitales internes et la paralysie de la mère ont obligé à pratiquer une césarienne avec sacrifice de la mère; le poulain quoique parfaitement constitué et à terme était mort.

\section{ACCIDENTS DE FOURBURE DUS EU SURRE}

Un troupeau de quarante-quatre chevaux indigènes dont les antécédents étaient inconnus a été soumis à une marche de 80 à 90 kilomètres en quarante-huit heures. Sur ce troupeau que nous avons visité, par hasard, à la fin du trajet, onze chevaux ont été atteints de fourbure aiguë des quatre membres en cours de route; les convoyeurs les avaient amenés au lieu de rassemblement avec un retard d'un jour. Lors de la visite sanitaire, à l'examen du sang périphérique entre lame et lamelle, tous les animaux atteints de fourbure aiguë présentaient une forte fièvre et des trypanosomes nombreux dans le sang périphérique. Il a été possible de déceler des trypanosomes dans le sang de deux autres animaux non atteints de fourbure, mais en mauvais état. Comme ce troupeau appartenait à des formations que nous ne pouvions contrôler qu'accidentellement, et avec de grandes difficultés, les malades ont été stérilisés au Naganol avec $1 \mathrm{gr}$. 50 en intraveineuse selon la méthode habituelle, mis en fourrière et vendus par la suite.

\section{CONCLUSION}

Au Cambodige, on peut assister sur les bovidés à des formes foudroyantes de surra, particulièrement sur les animaux reproducteurs pratiquant la monte régulièrement.

Les signes morbides de'surra chez les femelles bovines se manifestent plus particulièrement chez les animaux ayant un rendement plus élevé, que l'exportation phospho-calcique de la mamelle met en état de moindre résistance.

La mise en évidence des trypanosomes dans le sang périphérique par l'examen à l'état frais entre lame et lamelle est pratiquement très difficile.

Les symptômes observés ont quelques analogies avec le barbone; à l'autopsie, les œdèmes gélatineux infiltrés de liquide rose citrin peuvent prêter à confusion; on les differencie toujours des tumeurs lardacées du charbon bactéridien. Si la rate est congestionnée, elle ne peut prêter à confusion avec une rate d'animal charbonneux.

Le traitement au Naganol stérilise les bovidés de 250 kilos d'une façon qui paraît définitive avec deux injections de 3 grammes en solution au dixième en intraveineuse ou en intramusculaire.

Pour les équidés, le traitement au Naganol voit ses chances de succès très réduites pour les animaux ayant une forte proportion de sang importé. Ces chances sont encore plus minimes chez les étalons pratiquant la monte et les juments en gestation. Les trypanosomes disparaissent toujours du sang périphérique après la première injection, mais l'observation de longue durée montre que beaucoup d'animaux, en apparence guéris, ont des rechutes pour des causes diverses. Un temps d'observation de trois ans n'est pas superflu. L'utilisation des animaux ayant eu des atteintes de surra et traités au Naganol exige des ménagements et une surveillance qui diminue grandement les services rendus. A part quelques rares exceptions, les animaux sont perdus pour la reproduction. 